\title{
Liver function after transplantation in the assessment of the coagulation system and the concept of antithrombotic therapy
}

\author{
Marceli Lukaszewski ${ }^{1}$, Jacek Jakubaszko ${ }^{2}$, Grzegorz Bielicki ${ }^{2}$, Katarzyna Koscielska-Kasprzak ${ }^{3}$, Kinga Kosiorowska² \\ ${ }^{1}$ Department of Anaesthesiology and Intensive Therapy, Wroclaw Medical University, Wroclaw, Poland \\ 2Department of Cardiac Surgery, Wroclaw Medical University, Wroclaw, Poland \\ ${ }^{3}$ Department of Nephrology and Transplantation Medicine, Wroclaw Medical University, Wroclaw, Poland
}

Gastroenterology Rev 2020; 15 (1): 48-54

DOI: https://doi.org/10.5114/pg.2020.93631

Key words: anticoagulant therapy, anticoagulation, liver transplantation.

Address for correspondence: Marceli Lukaszewski MD, PhD, Wroclaw Medical University, Department of Anaesthesiology and Intensive Therapy, 213 Borowska St, 50-556 Wroclaw, Poland, phone: +48 7173323 10, fax: +48 71733 23 09, e-mail: marceliluk@gmail.com

\begin{abstract}
Introduction: Thrombotic complications after liver transplantation limit the long-term success of the procedure. Therefore, an early and accurate diagnosis with the appropriate treatment is crucial to sustain the proper functioning of the graft.

Aim: To evaluate the return of newly transplanted liver function within the first days of ICU stay after liver transplantation surgery (Ltx) observed in laboratory examination. It is important to understand the physiology of the newly transplanted liver, particularly in terms of its metabolic function and the assessment of easy-to-monitor coagulation parameters and enzyme markers.

Material and methods: We present our observations carried out in 27 patients, transplanted in the period 2015-2017, during their stay in the Department of Anaesthesiology and Intensive Therapy of the University Hospital in Wroclaw. We demonstrated changes in laboratory parameters within $72 \mathrm{~h}$ after liver transplantation and the concept of anticoagulant therapy at our institution.

Results: The presented results show the characteristics of aspartate transaminase, alanine transaminase, bilirubin, and standard tests evaluating the coagulation system within the first 4 days after surgery. The concept of anticoagulant therapy used in our intensive care unit is also presented. The aim of the work is an observation of physiology of the graft function in the aspect of coagulation disorders.

Conclusions: The early postoperative period is considered prognostic. The characteristics of basic biochemical tests are determined by the function of the transplanted organ. Implementation of anticoagulant therapy in this period is a therapeutic challenge that requires experience.
\end{abstract}

\section{Introduction}

The liver is a vital organ, and its dysfunction in liver cirrhosis leads to systemic disease, which not only affects metabolic and detoxification functions but also leads to respiratory or renal insufficiency. Because liver transplantation is currently an accepted first-line treatment, the procedure itself is burdened with many serious complications that may limit the long-term success of the procedure. The return of liver metabolic function after liver transplantation (LTX) expresses the proper functioning of vascular or biliary anastomoses and thus adequate organ perfusion. The main purpose of early graft assessment is to detect any type of liver dysfunction in the post-transplantation period. Early allograft dysfunction (EAD) is defined by the presence of at least one of the following: aspartate transaminase (AST) or alanine transaminase (ALT) above $2000 \mathrm{IU} / \mathrm{I}$ within the first week post LTx, total bilirubin $\geq 10 \mathrm{mg} /$ $\mathrm{dl}$, or international normalized ratio (INR) $\geq 1.6$ on the seventh postoperative day [1]. In the presence of EAD, the immediate implementation of therapy is essential.

\section{Coagulation system}

Coagulation disorders in end-stage liver disease and the early post-LTx period pose a serious therapeutic issue, and their pathology is complex and multifactorial. 
The major causes are a deficiency of either coagulation factors or anticoagulants, thrombocytopaenia due to platelet sequestration into the spleen and sinusoids, thrombocytopathy, or hyperfibrinolysis [2-5]. It is, therefore, crucial to sustain an effective assessment of coagulopathy with the appropriate use of anticoagulants. Although many factors, including surgical, may induce venous or hepatic artery thrombosis, coagulation disturbances may also play a crucial role in this process [6]. Since clinicians are aware of clotting complications and its various causes, the decision to implement antithrombotic therapy postoperatively appears to be reasonable. However, this decision is complex and still not regulated by any guidelines or recommendations. It is therefore extremely important to understand the physiology of the transplanted graft, particularly in terms of its metabolic function and the assessment of easy-to-monitor coagulation parameters and enzyme markers.

\section{Aim}

The aim of our study was to evaluate the return of the physiological function of the newly transplanted liver within the first days of intensive care unit (ICU) stay after LTx surgery.

\section{Material and methods}

We performed a retrospective analysis of patients following liver transplantation surgery during their ICU stay at Wroclaw University Hospital between 2015 and 2017. The patients' agreement for treatment and data collection was approved by the local Bioethics Committee. The dataset covering the early post-LTx period was collected from both electronic medical records and patients' charts. We analysed the age and sex of the patient, model of end-stage liver disease (MELD), indications for transplantation, the length of the ICU stay, as well as the coagulation status and haemodynamic changes before and after the operation. Haemodynamic monitoring was performed using a Swan-Ganz catheter. The laboratory tests were performed before and after the transplantation in the scheduled time intervals (Table I). All complications, especially those concerning impaired haemostasis, were also evaluated. Coagulation tests included measurement of prothrombin time (PT), activated partial thromboplastin time (APTT), fibrinogen concentration, antithrombin III (ATIII), and platelet count (PLT). Laboratory tests assessed also the activity of the main enzymes, e.g. AST, ALT, $\gamma$-glutamyltransferase (GGTP), and bilirubin. Chest X-ray was performed after ICU admission, while additional radiological investigations, such as computed tomography or visceral angiog- raphy, were performed if necessary. Doppler echoes were performed in the first and third day after the surgery in order to examine the flow in the graft vessels and detect eventual thrombosis or accumulation of fluid in the abdominal cavity, which could suggest active bleeding. We also observed the type and amount of blood products transfused and the implemented anticoagulant therapy in the following days of ICU stay after the procedure.

\section{Statistical analysis}

The obtained data were statistically analysed using Statistica 10.10 (StatSoft Inc.). Analysis of coagulation status was expressed by basic statistical concepts like arithmetic means, medians, and minimum and maximum values, taking into account the standard deviation. The groups were compared with $t$-test for normally distributed variables and Wilcoxon tests in other cases.

\section{Results}

Between 2015 and 2017, a total of 31 patients underwent deceased-donor liver transplantation surgery.

Table I. Schedule of the time intervals with $X$ indicating the measured parameter

\begin{tabular}{lc} 
X0 & Immediately before LTX \\
\hline X1 & Immediately after LTX \\
\hline X2 & 24 h post LTX \\
\hline X3 & 48 h post LTX \\
\hline X4 & 72 h post LTX
\end{tabular}

LTX - liver transplantation.

Table II. Patients' demographics with operative details

\begin{tabular}{lc} 
Parameter & Results \\
\hline Patients, $n$ & 27 \\
\hline Age, mean \pm SD [years] & $46.8 \pm 13.5$ \\
\hline Gender male, $n(\%)$ & $16(59.3)$ \\
\hline MELD, $n=26$, mean \pm SD & $15.6 \pm 5.0$ \\
\hline ICU stay, mean (min.-max.) [days] & $6.1(3-20)$ \\
\hline CIT, $n=26$, mean \pm SD [h] & $7.7 \pm 0.9$ \\
\hline WIT, $n=27$, mean \pm SD [h] & $51.9 \pm 9.2$ \\
\hline Operation time, mean \pm SD [h] & $8.1 \pm 1.4$ \\
\hline Intraoperative blood loss, $n=15$, mean \pm SD [ml] & $466.7 \pm 306.3$
\end{tabular}

MELD - model of end-stage liver disease, CIT - cold ischaemia time, WIT-warm ischaemia time. 
Of those, 4 (9.6\%) patients, who died due to primary liver failure, multi-organ failure following re-transplantation, or complications caused by portal vein thrombosis, were excluded from the study. In total, 27 patients (mean age: $46.8 \pm 13.5$ years, 16 males) following primary liver transplantation surgery were included in this retrospective study. Demographic findings, indica- tion for surgery, and operative details are presented in Table II. Equally one-third of liver cirrhosis causes were hepatitis and alcoholic cirrhosis; whereas, among the minor causes were primary sclerosis (PSC), autoimmune hepatitis (AIH), primary biliary cirrhosis, and Wilson's disease. The average MELD score in the preoperative period was 15 . The mean length of ICU stay was

Table III. Laboratory results of five core coagulation parameters for patients before and after liver transplantation

\begin{tabular}{|c|c|c|c|c|c|c|c|c|}
\hline Parameter & $N$ & Mean & Median & Min. & Max. & Low & High & SD \\
\hline \multicolumn{9}{|l|}{ PT (\%): } \\
\hline 0 & 27 & 73.8 & 74.7 & 29.7 & 100.5 & 65.7 & 85.4 & 14.5 \\
\hline 1 & 27 & 55.6 & 56.2 & 30.6 & 78.9 & 46.9 & 63.9 & 11.0 \\
\hline 2 & 26 & 65.8 & 63 & 48.8 & 86.3 & 58 & 73.5 & 10.7 \\
\hline 3 & 27 & 75.8 & 73.5 & 53.9 & 100.9 & 67.3 & 85.8 & 12.2 \\
\hline 4 & 26 & 81.6 & 80.3 & 63.1 & 100.8 & 72 & 92.7 & 11.3 \\
\hline \multicolumn{9}{|l|}{ APTT [s]: } \\
\hline 0 & 26 & 40.9 & 39.3 & 29.3 & 57.3 & 33.0 & 51.2 & 9.1 \\
\hline 1 & 27 & 79.2 & 74.4 & 40.5 & 126.7 & 55.7 & 103.0 & 25.8 \\
\hline 2 & 27 & 55.3 & 53.0 & 40.4 & 101.8 & 46.9 & 61.4 & 13.0 \\
\hline 3 & 27 & 50.1 & 50.3 & 33.5 & 67.8 & 40.5 & 58.5 & 9.3 \\
\hline 4 & 26 & 44.8 & 44.7 & 27.9 & 59.3 & 38.6 & 53.4 & 8.4 \\
\hline \multicolumn{9}{|c|}{$\operatorname{PLT}\left[\times 10^{3} / \mu \mathrm{l}\right]:$} \\
\hline 0 & 27 & 111.9 & 83.0 & 36.0 & 270.0 & 68.0 & 161.0 & 67.8 \\
\hline 1 & 27 & 139.0 & 100.0 & 39.0 & 391.0 & 76.0 & 191.0 & 88.5 \\
\hline 2 & 27 & 98.7 & 67.0 & 17.0 & 437.0 & 43.0 & 131.0 & 92.8 \\
\hline 3 & 27 & 55.1 & 43.0 & 12.0 & 244.0 & 24.0 & 65.0 & 50.2 \\
\hline 4 & 26 & 48.9 & 35.5 & 13.0 & 214.0 & 23.0 & 55.0 & 44.9 \\
\hline \multicolumn{9}{|c|}{ Fibrinogen $[\mathrm{g} / \mathrm{dl}]$ : } \\
\hline 0 & 24 & 2.4 & 2.3 & 0.0 & 5.7 & 1.5 & 2.9 & 1.2 \\
\hline 1 & 26 & 2.2 & 2.3 & 1.3 & 3.6 & 1.7 & 2.4 & 0.5 \\
\hline 2 & 22 & 2.5 & 2.4 & 1.6 & 3.5 & 2.3 & 2.8 & 0.5 \\
\hline 3 & 14 & 2.4 & 2.4 & 1.4 & 3.8 & 1.8 & 2.7 & 0.7 \\
\hline 4 & 14 & 2.2 & 2.0 & 1.5 & 3.5 & 1.9 & 2.6 & 0.6 \\
\hline \multicolumn{9}{|l|}{ ATIII (\%): } \\
\hline 0 & 25 & 51.9 & 42.5 & 17.9 & 102.0 & 33.7 & 63.9 & 24.9 \\
\hline 1 & 27 & 37.4 & 36.0 & 16.5 & 64.7 & 31.6 & 41.8 & 10.6 \\
\hline 2 & 20 & 39.8 & 40.3 & 17.9 & 67.2 & 34.2 & 44.7 & 10.7 \\
\hline 3 & 26 & 49.2 & 49.5 & 22.3 & 76.1 & 39.2 & 57.5 & 13.5 \\
\hline 4 & 20 & 60.2 & 58.5 & 36.9 & 87.4 & 50.5 & 68.1 & 14.1 \\
\hline
\end{tabular}


7.9 days. Detailed data of measured laboratory parameters performed within strictly scheduled time intervals are presented in Table III.

\section{Coagulation status}

Preoperative coagulation status analysis showed a mean PT value of $73.85 \%$, APTT of 40.95 s, fibrinogen of $2.44 \mathrm{~g} / \mathrm{dl}$, and ATIII level of $51.91 \%$. The platelets count ranged from $36 \times 10^{3} / \mu$ to $270 \times 10^{3} / \mu$ with average $110.5 \times 10^{3} / \mu$ l (Figure 1 ). Initial PLT count below $100 \times 10^{3} / \mu$ l was reported in $18(60 \%)$ patients. Of note, none of the patients underwent splenectomy prior to the transplantation. Three (11.1\%) patients admitted to the ICU required surgical intervention due to postoperative bleeding in the first postoperative day (POD1). In all patients, the PT immediately after LTx was slightly decreased with a mean of $55.6 \%$, but it gradually returned to a normal range within $72 \mathrm{~h}$ (Figure 2). The average APTT immediately after the procedure was $79 \mathrm{~s}$, and it tended to normalise within $24 \mathrm{~h}$ of ICU stay (Figure 3). In the absence of clinical or ultrasound features of active bleeding, platelets count above $20 \times 10^{3} / \mu \mathrm{l}$ with APTT within the therapeutic range, i.e. 40-60 s, was maintained by the infusion of unfractionated heparin (UFH) at the appropriate dose every 4-6 h. The UFH treatment was implemented for an average of 4.21 days. The thromboembolic prophylaxis was then followed by the use of low-molecular-weight heparins (LMWH). Mean platelet count in $48 \mathrm{~h}$ post LTx observation was $55 \times 10^{3} / \mu \mathrm{l}\left(13-214 \times 10^{3} / \mu \mathrm{l}\right)$. The triggers for substitution of platelets were the PLT lower than

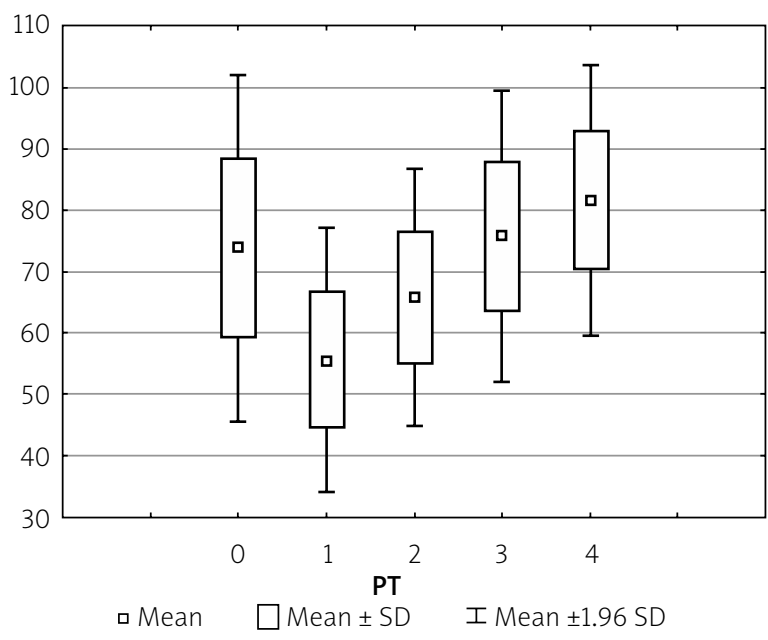

Figure 2. Measurements of prothrombin time (PT) performed within scheduled time intervals. Relations between measurements are statistically significant from $p<0.05$

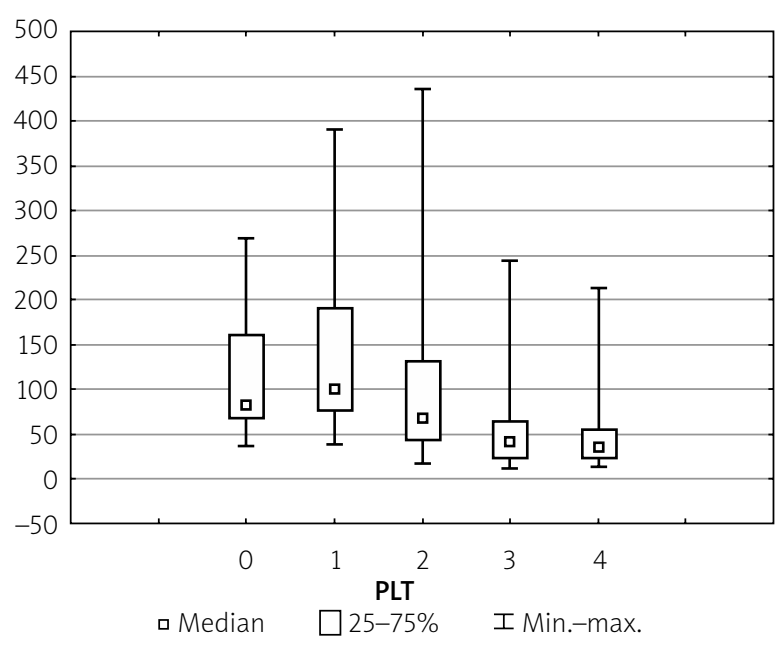

Figure 1. Boxplot illustrating the physiology of the newly transplanted liver represented by platelet count (PLT). Each vertical box describes the distribution of the measured laboratory values

$20 \times 10^{3} / \mu \mathrm{l}$ or observed clinical signs of active bleeding (Table IV). During the ICU observation the platelet count in the study group substantially decreased. Consequently, after $72 \mathrm{~h}$, in approximately $70 \%$ of patients, the platelet level was lower than $50 \times 10^{3} / \mu$ l whereas only in about $7 \%$ of patients the PLT was above $100 \times 10^{3} / \mu \mathrm{l}$ (Figure 1). The average level of fibrinogen after the LTX was $2.18 \mathrm{~g} / \mathrm{dl}$, and it remained stable for the subsequent 3 days (Figure 4). Together with normalisation of PT and fibrinogen, levels of ATIII dropped significantly below $80 \%$ (Figure 5).

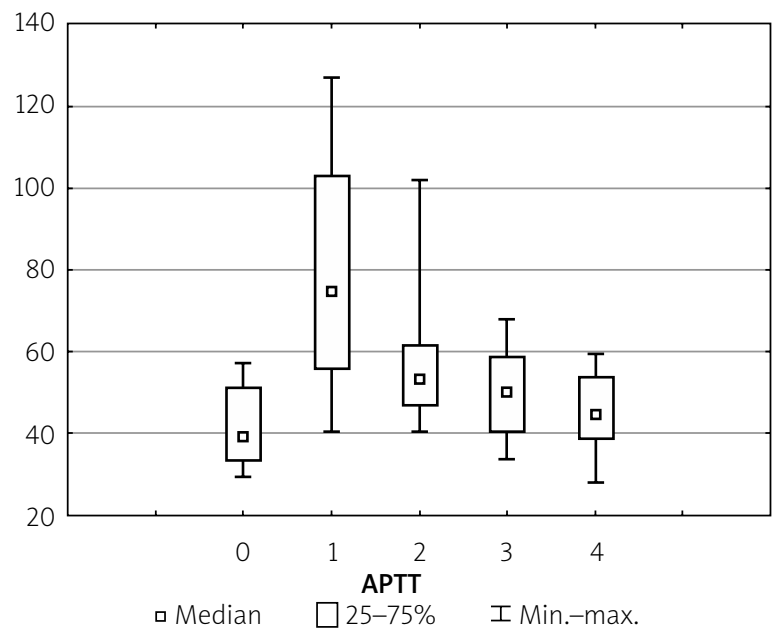

Figure 3. Boxplot illustrating the physiology of the newly transplanted liver represented by partial thromboplastin time after activation (APTT). Each vertical box describes the distribution of the measured laboratory values 
Table IV. Transfusion products supplemented in patients with bleeding complication

\begin{tabular}{lc}
\hline Parameter & Results \\
\hline Bleeding patients, $n(\%)$ & $3(11.1)$ \\
\hline PRBC [U] & 5 \\
\hline ATIII [U] & 8 \\
\hline FFP [U] & 4 \\
\hline PPC [U] & 0 \\
\hline Fibrinogen $[g]$ & 2 \\
\hline PLT [U] & 3
\end{tabular}

\section{Enzyme levels}

Levels of liver markers follow a very similar trend during the whole postoperative course (Figures 6-8). After the initial increase in values of AST, ALT, and bilirubin within 2 days of observation there was a slight decrease noted in the following period. Thirteen (48.1\%) patients developed transient EAD, of whom 9 (33.3\%) had increased levels of AST up to $1000 \mathrm{U}$ and 4 (14.8\%) patients presented with increased levels of AST up to $3000 \mathrm{U}$. Nevertheless, none of those patients required the substitution of coagulation factors. Furthermore, AST and ALT levels were normalised within the following week of observation.

\section{Discussion}

In the study, because we focus on the core biochemical laboratory parameters, derivates of coagulation,

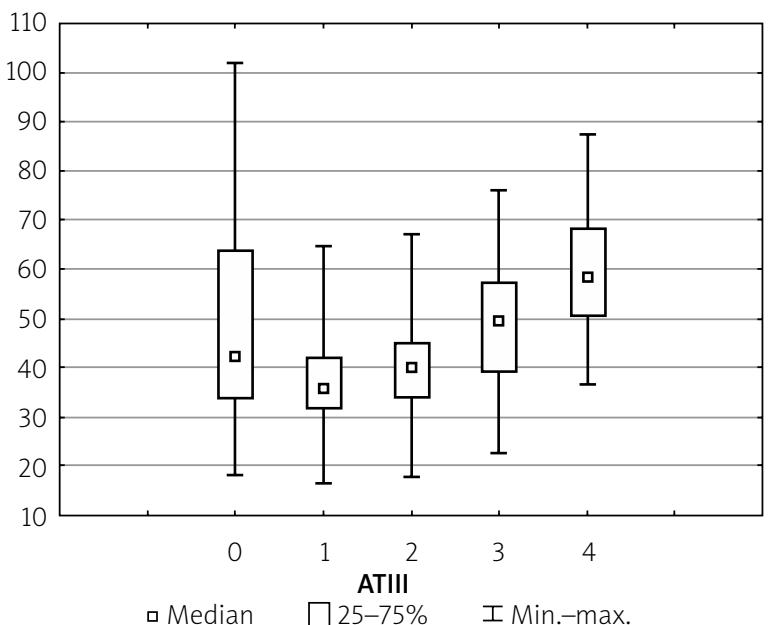

Figure 5. Boxplot illustrating the physiology of the newly transplanted liver represented by antithrombin III (ATIII). Each vertical box describes the distribution of the measured laboratory values

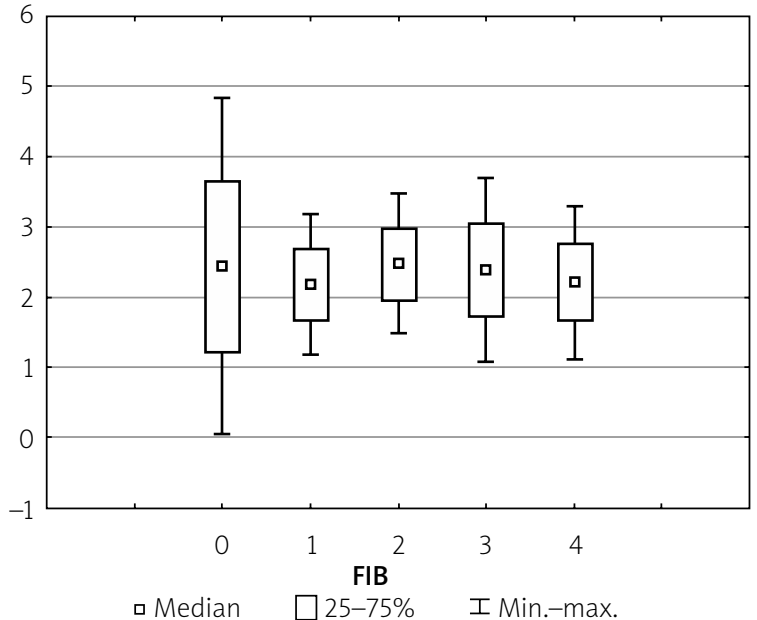

Figure 4. Boxplot illustrating the physiology of the newly transplanted liver represented by fibrinogen. Each vertical box describes the distribution of the measured laboratory values

we demonstrate physiological changes that occur in the newly transplanted liver within the first $72 \mathrm{~h}$ after transplantation. The parameters evaluating the coagulation system that were taken into account were APTT, PT, ATIII, and platelet count. Coagulopathy is a severe complication after liver transplantation that limits the long-term success of the entire procedure. Therefore, knowing the exact timing of anticoagulant implementation is crucial. In our observational group, when APTT tended to normalise and in the absence of active bleeding confirmed by echo and laboratory examinations, an

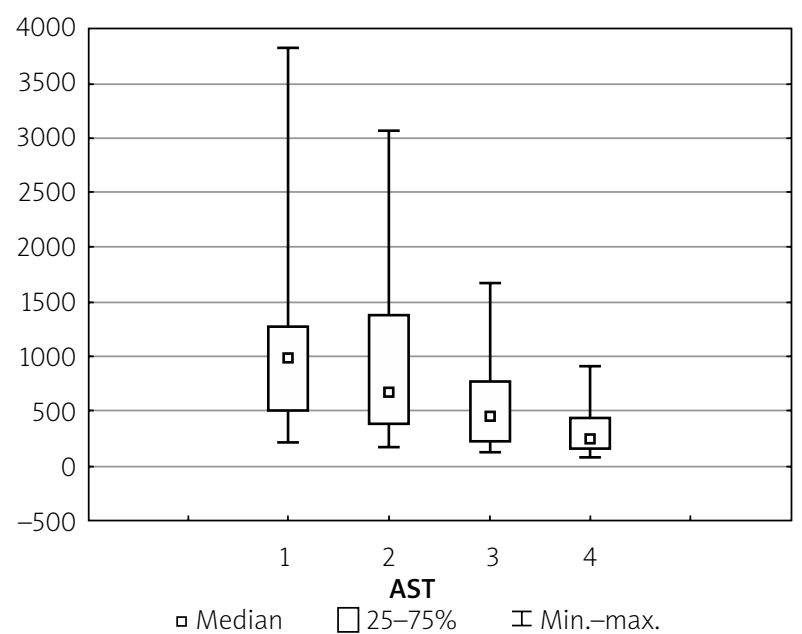

Figure 6. Boxplot illustrating the physiology of the newly transplanted liver represented by aspartate transaminase (AST). Each vertical box describes the distribution of the measured laboratory values 


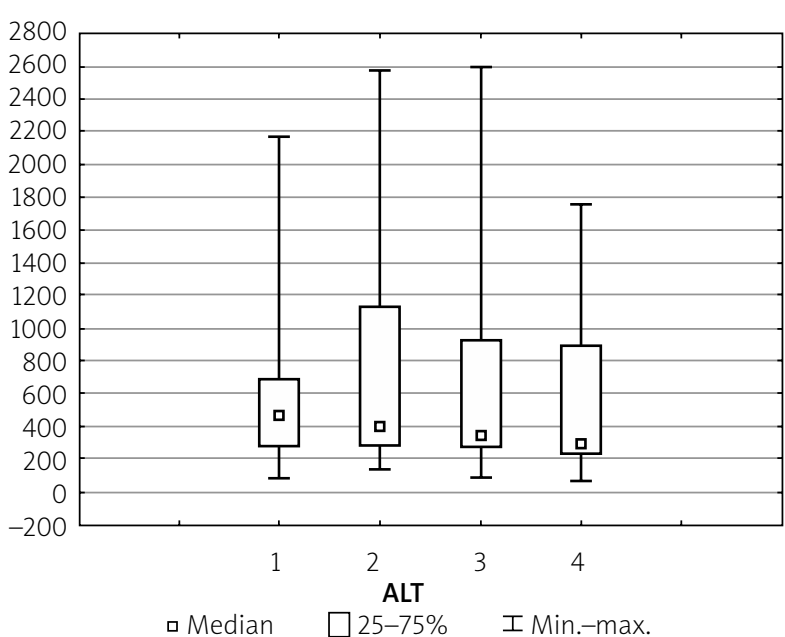

Figure 7. Boxplot illustrating the physiology of the newly transplanted liver represented by alanine transaminase (ALT). Each vertical box describes the distribution of the measured laboratory values

infusion of UFH within the therapeutic range (i.e. APTT 40-60 s) was initiated [7]. Typically, heparin infusion was started on POD1 and continued for an average of 4 days, followed by implementation of low-molecular-weight heparin in anticoagulant prophylaxis. In the ICU observation, apart from three patients with active bleeding immediately after the surgery, no cases of haemorrhagic complications were reported. In those patients who developed bleeding, the heparin infusion was discontinued, and the antithrombotic therapy was resumed only after cessation of bleeding. Undoubtedly, antithrombotic treatment after LTX is an important clinical issue. In many observations, the return of liver function, in the aspect of the synthesis of clotting factors, disturbs the delicate balance between pro- and anticoagulants. Because there is a disproportionately greater production of prothrombotic agents compared to anticoagulants such as ATIII, protein S, and protein C, a hypercoagulable phenomenon in the early post-LTX period is frequently initiated. Similarly, in our observations, the synthesis of prothrombotic agents proceeds asymmetrically to the synthesis of coagulation inhibitors (Table III) [8, 9]. Antithrombotic therapy through requires adequate adjustment of the heparin dose (usually $3-5 \mathrm{U} / \mathrm{kg} / \mathrm{h}$ ), and in some cases also ATIII substitution. Nearly $30 \%$ of the described group of patients needed ATIII substitution to achieve the effective action of heparin and the expected prolongation of APTT until 1.5 times the normal value (Table IV). Due to the lack of clear guidelines, transplant centres have established their own protocols based on many years of experience.

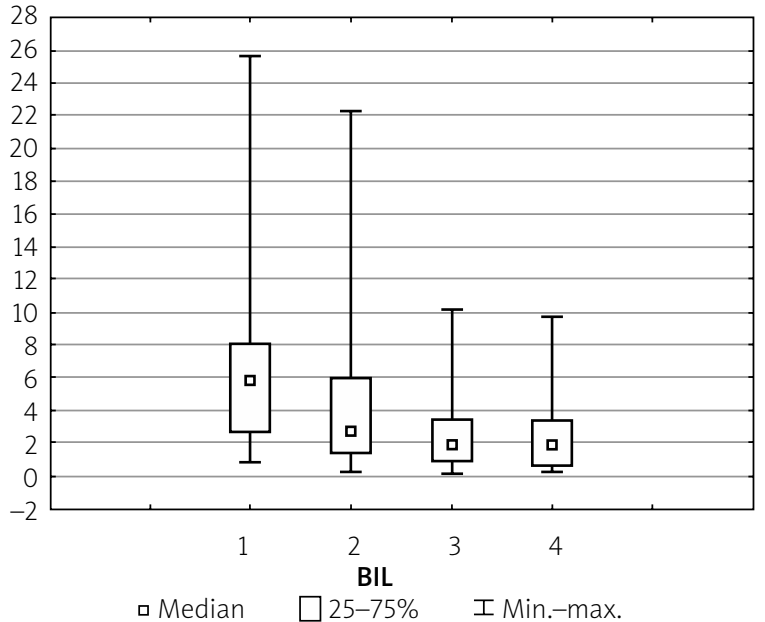

Figure 8. Boxplot illustrating the physiology of the newly transplanted liver represented by bilirubin. Each vertical box describes the distribution of the measured laboratory values

Undoubtedly, one of the most controversial issues is the use of anticoagulation therapy post LTX. However, with the current state of knowledge and familiarity with the physiology of the graft, many authors are inclined to use the infusion of UFH followed by LMWH [8]. In regard to platelet count, there was a substantial decrease in the PLT observed within 3 days after surgery with 19 patients having a PLT below $50 \times 10^{3} / \mu$ lat the end of ICU stay. Furthermore, some research confirms our observation that in patients after liver transplantation the platelet count is often reduced because of entrapment in the sinusoids in the donor's liver [10]. Additionally, thrombocytopaenia in cirrhosis with high levels of von Willebrand factor (vWF) is well compensated and balanced [11]. However, the time for which this effect persists after liver transplantation is not known. According to the standard of our department, platelet transfusion is performed either if the PLT is below $20 \times 10^{3} / \mu$ or in the case of clinical manifestations of active bleeding. However, some authors claim that prophylactic use of platelets should be avoided because higher platelet counts may be detrimental [12]. Platelets transfusions are believed to contribute to thrombotic complications and an increase in mortality after liver transplantation $[13,14]$. In regard to $R B C$, the indication for transfusion was haemoglobin level lower than $7 \mathrm{~g} / \mathrm{dl}$ or active excessive bleeding [15]. Research reports that intraoperative transfusions of PRBC and PLT are an independent risk factor for survival after LTx and thus inevitably increase mortality [16]. After liver transplantation, both pro- and anticoagulant pathways are impaired. The 
balance of the coagulation system in the early postoperative period changes rapidly as the transplanted organ takes on its function. By the time a new balance is established, the patient is exposed to many serious complications such as postoperative bleeding or thrombosis. Therefore, the understanding of the graft physiology after transplantation may allow a bespoke decision to be made about precise therapy in order to avoid more serious post-transplantation complications. It is also extremely important to maintain clinical and biochemical markers of a properly functioning liver within the range of accepted values for at least a few days after LTx. Unfortunately, standard coagulation laboratory tests and the platelet count do not provide complete information about the coagulation status. Therefore, it seems that global coagulation tests such as ROTEM or TEG may be beneficial for patients after LTx [17]. We believe that in light of current knowledge, anticoagulant therapy should be implemented after every LTx equally, as in other complicated procedures. Current advances in surgical technique and the change in post-transplantation protocol without usage of transfusion and blood products may lead to the more frequent use of antithrombotic therapy, the inclusion of which appears to be essential.

\section{Conflict of interest}

The authors declare no conflict of interest.

\section{References}

1. Hudcova J, Scopa C, Rashid J, et al. Effect of early allograft dysfunction on outcomes following liver transplantation. Clin Transplant 2017; 31. doi: 10.1111/ctr.12887.

2. Feltracco P, Barbieri S, Galligioni H, et al. Intensive care management of liver transplanted patients. World J Hepatol 2011; 3: 61-71.

3. Hartmann M, Szalai C, Saner FH. Hemostasis in liver transplantation: pathophysiology, monitoring, and treatment. World J Gastroenterol 2016; 22: 1541-50.

4. Mallett SV, Chowdary P, Burroughs AK. Clinical utility of viscoelastic tests of coagulation in patients with liver disease. Liver Int 2013; 33: 961-74.

5. Balzer F, Sander M, Simon M, et al. High central venous saturation after cardiac surgery is associated with increased organ failure and long-term mortality: an observational cross-sectional study. Crit Care 2015; 19: 168.

6. Stahl RL, Duncan A, Hooks MA, et al. A hypercoagulable state follows orthotopic liver transplantation. Hepatology 1990; 12: 553-8.

7. Algarni AA, Mourad MM, Bramhall SR. Anticoagulation and antiplatelets as prophylaxis for hepatic artery thrombosis after liver transplantation. World J Hepatol 2015; 7: 1238-43.

8. De Pietri L, Montalti L, Nicolini D, et al. Perioperative thromboprophylaxis in liver transplant patients. World I Gastroenterol 2018; 24: 2931-48.
9. Stahl RL, Duncan A, Hooks MA, et al. A hypercoagulable state follows orthotopic liver transplantation. Hepatology 1990; 12: 553-8.

10. Clevenger B, Mallett SV. Transfusion and coagulation management in liver transplantation. World J Gastroenterol 2014; 20 : 6146-58.

11. Lisman T, Bongers TN, Adelmeijer J, et al. Elevated levels of von Willebrand factor in cirrhosis support platelet adhesion despite reduced functional capacity. Hepatology 2006; 44: 53-61.

12. Gopal P, Kapoor D, Raya R, et al. Critical care issues in adult liver transplantation. Indian J Crit Care Med 2009; 13: 113-9.

13. Pereboom ITA, De Boer MT, Haagsma EB, et al. Platelet transfusion during liver transplantation is associated with increased postoperative mortality due to acute lung injury. Anesth Analg 2009; 108: 1083-91.

14. Pereboom ITA, Lisman T, Porte RJ. Platelets in liver transplantation: friend or foe? Liver Transplant 2008; 14: 923-31.

15. McIntyre L, Tinmouth LT, Fergusson DA. Blood component transfusion in critically ill patients. Curr Opin Crit Care 2013; 19: 326-33.

16. De Boer MT, Christensen MC, Asmussen M, et al. The impact of intraoperative transfusion of platelets and red blood cells on survival after liver transplantation. Anesth Analg 2008; 106: 32-44.

17. Zamper RPC, Amorim TC, da Costa LGV, et al. The role of thromboelastometry in the assessment and treatment of coagulopathy in liver transplant patients. Einstein 2017; 15: 243-6.

Received: 10.04 .2019

Accepted: 2.05 .2019 